\title{
Errata to "On Defining Relations of Affine Lie Superalgebras and Affine Quantized Universal Enveloping Superalgebras"
}

\author{
By
}

\author{
Hiroyuki YAMANE*
}

The paper [1] contains mistakes; Theorems 4.1.1, 4.5.1 and 8.4.3 for the data corresponding to the Dynkin diagrams below are incorrect.
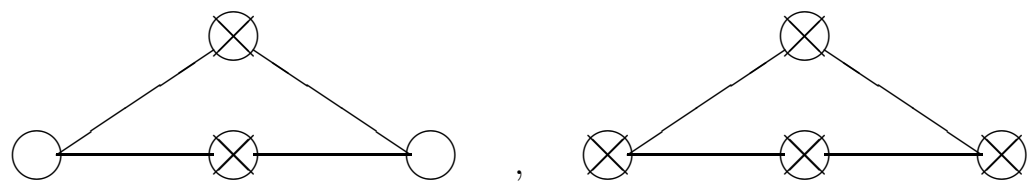

In Section 2, the statements and proofs of the theorems shall be corrected.

1 Preliminary. Keep the notation and terminology of [1]. We say that the datum $(\mathcal{E}, \Pi, p)$ is of $\left(\mathrm{A}(1,1)^{(1)}\right)^{\mathcal{H}}$ type if $(\mathcal{E}, \Pi, p)$ is of affine ABCD type (see Definition 1.4.1), $\Pi=\left\{\alpha_{0}, \alpha_{1}, \alpha_{2}, \alpha_{3}\right\}$ and if the Dynkin diagram of $(\mathcal{E}, \Pi, p)$ is either of the two Dynkin diagrams of (1). Until the end of this section, we assume that $(\mathcal{E}, \Pi, p)$ is of $\left(\mathrm{A}(1,1)^{(1)}\right)^{\mathcal{H}}$ type. Then $\mathcal{G}(\mathcal{E}, \Pi, p) \cong\left(\mathrm{A}(1,1)^{(1)}\right)^{\mathcal{H}}$, $\mathcal{G}^{\natural}(\mathcal{E}, \Pi, p) \cong\left(\operatorname{sl}(2,2)^{(1)}\right)^{\mathcal{H}}$, and $\Phi(\mathcal{E}, \Pi, p)=\left\{ \pm(m+1) \delta, \pm\left(m \delta+\alpha_{i}\right), \pm(m \delta+\right.$ $\left.\left.\alpha_{i}+\alpha_{i-1}\right), \pm\left(m \delta+\alpha_{i}+\alpha_{i-1}+\alpha_{i-2}\right) \mid i=0,1,2,3 \in \mathbf{Z} / 4 \mathbf{Z}, m \geq 0\right\}$. (See Subsections 1.5 and 3.5 , and notice that $\left(\mathrm{A}(1,1)^{(1)}\right)^{\mathcal{H}}$ and $\mathrm{A}(1,1)^{(1)}$ (resp. $\left(s l(2,2)^{(1)}\right)^{\mathcal{H}}$ and $\left.s l(2,2)^{(1)}\right)$ are not the same; however they are closely related.) Define $E_{i}^{(m)}, E_{i i-1}^{(m)}, E_{i i-1 i-2}^{(m)} \in \widetilde{\mathcal{N}}_{+}(\subset \widetilde{\mathcal{G}}(\mathcal{E}, \Pi, p))(i \in \mathbf{Z} / 4 \mathbf{Z}, m \geq 0)$ inductively by $E_{i}^{(0)}=E_{i}, E_{i}^{(m)}=\left[E_{i},\left[E_{i-1}, E_{i-2 i-3 i}^{(m-1)}\right], E_{i i-1}^{(m)}=\left[E_{i}, E_{i-1}^{(m)}\right], E_{i i-1 i-2}^{(m)}=\right.$ $\left[E_{i}, E_{i-1 i-2}^{(m)}\right]$. Let $E_{i i-1}=E_{i i-1}^{(0)}$ and $E_{i i-1 i-2}=E_{i i-1 i-2}^{(0)}$.

Let $\mathbf{x}: \widetilde{\mathcal{N}}_{+} \rightarrow \widetilde{\mathcal{N}}_{-}$be the isomorphism such that $\mathbf{x}\left(E_{i}\right)=F_{i}(0 \leq i \leq 3)$. Denote $(\mathcal{E}, \Pi, p)$ by $(\mathrm{AA})^{\star}$ if its Dynkin diagram is the left one of (1) and if

Communicated by M. Kashiwara, January 5, 2001.

2000 Mathematics Subject Classification(s): 17B37, 16W30, 17B70, 17B65.

* Department of Mathematics, Osaka University, Toyonaka 560-0043, Japan.

e-mail: yamane@math.sci.osaka-u.ac.jp 
$\left(\alpha_{1}, \alpha_{1}\right)=2$. Define $\mathcal{G}^{\star}$ to be the admissible Lie superalgebra $\widetilde{\mathcal{G}}(\mathrm{AA})^{\star} /\left(r_{+}^{\star}+\right.$ $\mathbf{x}\left(r_{+}^{\star}\right)$ ), where $r_{+}^{\star}$ is the ideal of $\widetilde{\mathcal{N}}_{+}$generated by the elements:

$$
\left\{\begin{aligned}
& {\left[E_{0}, E_{0}\right],\left[E_{2}, E_{2}\right],\left[E_{0}, E_{2}\right],\left[E_{1}, E_{3}\right], } \\
& {\left[E_{1}, E_{10}\right],\left[E_{1}, E_{21}\right],\left[E_{3}, E_{03}\right],\left[E_{3}, E_{32}\right], } \\
& {\left[E_{2}, E_{321}\right],\left[E_{210}, E_{032}\right],\left[E_{2},\left[E_{0}, E_{103}\right]\right] . }
\end{aligned}\right.
$$

(See Definition 1.2.1 for the terminology.) Denote $(\mathcal{E}, \Pi, p)$ by $(\mathrm{AA})^{\bullet}$ if its Dynkin diagram is the right one of $(1)$ and if $\left(\alpha_{1}, \alpha_{2}\right)=1$. Define $\mathcal{G}^{\bullet}$ to be the admissible Lie superalgebra $\widetilde{\mathcal{G}}(\mathrm{AA})^{\bullet} /\left(r_{+}^{\bullet}+\mathbf{x}\left(r_{+}^{\bullet}\right)\right)$, where $r_{+}^{\bullet}$ is the ideal of $\widetilde{\mathcal{N}}_{+}$ generated by the elements:

$$
\left\{\begin{array}{l}
{\left[E_{0}, E_{0}\right],\left[E_{1}, E_{1}\right],\left[E_{2}, E_{2}\right],\left[E_{3}, E_{3}\right],\left[E_{0}, E_{2}\right],\left[E_{1}, E_{3}\right],} \\
{\left[E_{0}, E_{103}\right],\left[E_{1}, E_{210}\right],\left[E_{2}, E_{321}\right],\left[E_{3}, E_{032}\right] .}
\end{array}\right.
$$

Lemma 1. Let $(\mathcal{E}, \Pi, p)=(\mathrm{AA})^{\bullet}$, and $C^{\bullet}:=\left\{ \pm\left(m \delta+\alpha_{i}+\alpha_{i-2}\right) \mid i \in\right.$ $\mathbf{Z} / 4 \mathbf{Z}, m \geq 1\}$. Then:

(1) $\operatorname{dim} \mathcal{G}_{\beta}^{\bullet}=1$ if $\beta \in \Phi(\mathcal{E}, \Pi, p) \backslash \mathbf{Z} \delta$.

(2) $\operatorname{dim} \mathcal{G}_{\beta}^{\bullet}=0$ if $\beta \in\left(P_{+} \cup P_{-}\right) \backslash\left(\Phi(\mathcal{E}, \Pi, p) \cup C^{\bullet} \cup\{0\}\right)$.

(3) $\mathcal{G}_{\beta}^{\bullet}=\left[E_{i}, \mathcal{G}_{\beta-\alpha_{i}}^{\bullet}\right], \mathcal{G}_{-\beta}^{\bullet}=\left[F_{i}, \mathcal{G}_{-\beta+\alpha_{i}}\right]$ if $\beta=m \delta+\alpha_{i}+\alpha_{i-2}$ with $m \geq 1$.

Proof. Let a be the automorphism of $\mathcal{G} \bullet$ such that $\mathbf{a}\left(E_{i}\right)=E_{i-2}, \mathbf{a}\left(F_{i}\right)=$ $F_{i-2}$. Let $\mathbf{b}_{i}:=\exp \left(\operatorname{ad}\left[E_{i}, E_{i-1}\right]\right) \exp \left(\operatorname{ad}\left[F_{i}, F_{i-1}\right]\right) \exp \left(\operatorname{ad}\left[E_{i}, E_{i-1}\right]\right)$, and $\mathbf{c}_{i}:=$ $\mathbf{a} \circ \mathbf{b}_{i} \circ \mathbf{b}_{i+1}$. (Notice that $\operatorname{ad}\left[E_{i}, E_{i-1}\right]$ and $\operatorname{ad}\left[F_{i}, F_{i-1}\right]$ are locally nilpotent.) If $\beta=a_{0} \alpha_{0}+a_{1} \alpha_{1}+a_{2} \alpha_{2}+a_{3} \alpha_{3}$, then $\mathbf{c}_{i}\left(\mathcal{G}_{\beta}^{\bullet}\right)=\mathcal{G}_{\beta-\left(a_{i}-a_{i-2}\right) \delta}^{\bullet}$.

Notice that $\mathcal{G}^{\bullet}$ has a triangular decomposition $\mathcal{G}^{\bullet}=\mathcal{N}_{+}^{\bullet} \oplus \mathcal{H} \oplus \mathcal{N}_{-}^{\bullet}$, where $\mathcal{N}_{+}^{\bullet}=\tilde{\mathcal{N}}_{+} / r_{+}^{\bullet}$ and $\mathcal{N}_{-}^{\bullet}=\widetilde{\mathcal{N}}_{-} / \mathbf{x}\left(r_{+}^{\bullet}\right)$. Assume that $\beta \in P_{+} \backslash\{0\}$ and that we have already proved the lemma for $\beta^{\prime} \in P_{+} \backslash\{0\}$ with $\beta-\beta^{\prime} \in P_{+} \backslash\{0\}$. Then the lemma can be proved as follows; here we shall treat two cases as examples. First example is the case where $\beta=m \delta+\alpha_{i+1}+2 \alpha_{i}+\alpha_{i-1}$ with $m \geq 0$. If $m$ is even, it follows that $\operatorname{dim} \mathcal{G}_{\beta}^{\bullet}=\operatorname{dim} \mathbf{c}_{i}^{m / 2}\left(\mathcal{G}_{\beta}^{\bullet}\right)=\operatorname{dim} \mathcal{G}_{\alpha_{i+1}+2 \alpha_{i}+\alpha_{i-1}}^{\bullet}=$ $\operatorname{dim} \mathcal{N}_{+}^{\bullet} \cap \mathcal{G}_{\alpha_{i+1}+2 \alpha_{i}+\alpha_{i-1}}^{\bullet}=0$, where the last equality can be proved directly. If $m$ is odd, it follows that $\operatorname{dim} \mathcal{G}_{\beta}^{\bullet}=\operatorname{dim} \mathcal{G}_{\alpha_{i}-\alpha_{i-2}}^{\bullet}=0$. Second example is the case where $\beta=m \delta+\alpha_{i}+\alpha_{i-2}$ with $m \geq 0$. By the assumption, we have $\mathcal{G}_{\beta}^{\bullet}=\sum_{j \in \mathbf{Z} / 4 \mathbf{Z}}\left[E_{j}, \mathcal{G}_{\beta-\alpha_{j}}^{\bullet}\right]=\left[E_{i}, \mathcal{G}_{m \delta+\alpha_{i-2}}^{\bullet}\right]+\left[E_{i-2}, \mathcal{G}_{m \delta+\alpha_{i}}^{\bullet}\right]=$ $\left[E_{i},\left[E_{i-2}, \mathcal{G}_{m \delta}^{\bullet}\right]\right]+\left[E_{i-2},\left[E_{i}, \mathcal{G}_{m \delta}^{\bullet}\right]\right]=\left[E_{i},\left[E_{i-2}, \mathcal{G}_{m \delta}^{\bullet}\right]\right]=\left[E_{i}, \mathcal{G}_{m \delta+\alpha_{i-2}}^{\bullet}\right]$. Other cases can be treated similarly.

The case where $\beta \in P_{-} \backslash\{0\}$ can also be treated similarly. 
Lemma 2. Let $(\mathcal{E}, \Pi, p)=(\mathrm{AA})^{\star}$, and $C^{\star}:=\left\{ \pm\left((m+1) \delta+\alpha_{3}+2 \alpha_{2}+\right.\right.$ $\left.\left.\alpha_{1}\right), \pm\left(m \delta+\alpha_{1}+2 \alpha_{0}+\alpha_{3}\right) \mid m \geq 0\right\}$. Then:

(1) $\operatorname{dim} \mathcal{G}_{\beta}^{\star}=1$ if $\beta \in \Phi(\mathcal{E}, \Pi, p) \backslash \mathbf{Z} \delta$.

(2) $\operatorname{dim} \mathcal{G}_{\beta}^{\star}=0$ if $\beta \in\left(P_{+} \cup P_{-}\right) \backslash\left(\Phi(\mathcal{E}, \Pi, p) \cup C^{\star} \cup\{0\}\right)$.

(3) $\mathcal{G}_{\beta}^{\star}=\left[E_{i}, \mathcal{G}_{\beta-\alpha_{i}}^{\star}\right], \mathcal{G}_{-\beta}^{\star}=\left[F_{i}, \mathcal{G}_{-\beta+\alpha_{i}}^{\star}\right]$ if $\beta=m \delta+\alpha_{i+1}+2 \alpha_{i}+\alpha_{i-1}$ with $m \geq 0$.

Proof. Recall the definition of $s_{2}$ from Proposition 2.4.3. We can easily show that there exists an isomorphism $\mathbf{e}: \mathcal{G}^{\bullet} \rightarrow \mathcal{G}^{\star}$ such that $\mathbf{e}\left(H_{\gamma}\right)=H_{s_{2}(\gamma)}$, $\mathbf{e}\left(E_{j}\right)=-\left(\alpha_{j}, \alpha_{2}\right)\left[E_{j}, E_{2}\right], \mathbf{e}\left(F_{j}\right)=-\left[F_{j}, F_{2}\right](j=1,3), \mathbf{e}\left(E_{2}\right)=F_{2}, \mathbf{e}\left(F_{2}\right)=$ $-E_{2}, \mathbf{e}\left(E_{0}\right)=E_{0}, \mathbf{e}\left(F_{0}\right)=F_{0}$. Then the statements (1) and (2) follow from Lemma 1 and from the fact that $\mathbf{e}\left(\mathcal{G}_{\alpha}^{\bullet}\right)=\mathcal{G}_{s_{2}(\alpha)}^{\star}$. The statement (3) can be proved by an exactly similar argument to that for Lemma 1 (3).

Keep the notation of Definition 1.2.1. By the fact that $\mathcal{G}^{\natural}(\mathrm{AA})^{\star} \cong$ $\left(\operatorname{sl}(2,2)^{(1)}\right)^{\mathcal{H}}$, we can easily see that $\mathcal{G}^{\star} \succ \mathcal{G}^{\natural}(\mathrm{AA})^{\star}$. Hence $\Psi\left[\mathcal{G}^{\star}, \mathcal{G}^{\natural}(\mathrm{AA})^{\star}\right]$ can be defined. Similarly $\Psi\left[\mathcal{G}^{\bullet}, \mathcal{G}^{\natural}(\mathrm{AA})^{\bullet}\right]$ can be defined. Let $\Psi^{\star \natural}=\Psi\left[\mathcal{G}^{\star}, \mathcal{G}^{\natural}(\mathrm{AA})^{\star}\right]$, $\Psi^{M \star}=\Psi\left[\widetilde{\mathcal{G}}(\mathrm{AA})^{\star}, \mathcal{G}^{\star}\right], \Psi^{\bullet \natural}=\Psi\left[\mathcal{G}^{\bullet}, \mathcal{G}^{\natural}(\mathrm{AA})^{\bullet}\right]$ and $\Psi^{M \bullet}=\Psi\left[\widetilde{\mathcal{G}}(\mathrm{AA})^{\bullet}, \mathcal{G}^{\bullet}\right]$.

Theorem 3. (1) The ideal ker $\Psi^{\star \natural}$ is generated by the elements $\Psi^{M \star}$ $\left(\left[E_{0}, E_{103}^{(m)}\right]\right), \Psi^{M \star}\left(\left[E_{2}, E_{321}^{(m+1)}\right]\right), \quad \Psi^{M \star}\left(\mathbf{x}\left(\left[E_{0}, E_{103}^{(m)}\right]\right)\right), \quad \Psi^{M \star}\left(\mathbf{x}\left(\left[E_{2}, E_{321}^{(m+1)}\right]\right)\right)$ $(m \geq 0)$.

(2) The ideal ker $\Psi^{\bullet}$ is generated by the elements $\Psi^{M \bullet}\left(\left[E_{i}, E_{i+2}^{(m)}\right]\right), \Psi^{M \bullet}$ $\left(\mathbf{x}\left(\left[E_{i}, E_{i+2}^{(m)}\right]\right)\right)(i=0,1, m \geq 1)$.

Proof. Let $m \geq 0$, and let $Y \in \widetilde{\mathcal{G}}(\mathrm{AA})^{\star}$ be $E_{321}^{(m+1)}, E_{103}^{(m)}, \mathbf{x}\left(E_{321}^{(m+1)}\right)$ or $\mathbf{x}\left(E_{103}^{(m)}\right)$. Let $\gamma \in P$ be such that $Y \in \widetilde{\mathcal{G}}_{\gamma}$. By the fact that $\mathcal{G}^{\natural}(\mathrm{AA})^{\star} \cong$ $\left(\operatorname{sl}(2,2)^{(1)}\right)^{\mathcal{H}}$, we see that $\Psi^{\star \star} \circ \Psi^{M \star}(Y) \neq 0$. It follows from Lemma 2 (1) that $\mathcal{G}_{\gamma}^{\star}$ is the one dimensional vector space spanned by $\Psi^{M \star}(Y)$. Then the statement (1) follows immediately from Lemma 2. (We see that since $\mathcal{G}^{\natural}(\mathrm{AA})^{\star}$ is the maximal affine-admissible Lie superalgebra, $\operatorname{ker} \Psi^{\star \natural} \cap \mathcal{G}_{m \delta}^{\star}=\{0\}$ for $m \neq 0$.)

(2) We can show this by a similar argument to that for (1), and by Lemma 1.

We say that $(\mathcal{E}, \Pi, p)$ is a usual datum of $\left(\mathrm{A}(1,1)^{(1)}\right)^{\mathcal{H}}$ type, if $\left(\alpha_{i}, \alpha_{i}\right) \neq 0$ for some $0 \leq i \leq 3$. Otherwise we say that $(\mathcal{E}, \Pi, p)$ is an unusual datum of 
$\left(\mathrm{A}(1,1)^{(1)}\right)^{\mathcal{H}}$ type. If $(\mathcal{E}, \Pi, p)$ is a usual datum of $\left(\mathrm{A}(1,1)^{(1)}\right)^{\mathcal{H}}$ type and if $\left(\alpha_{1}, \alpha_{1}\right) \neq 0\left(\operatorname{resp} .\left(\alpha_{1}, \alpha_{1}\right)=0\right)$, let $\kappa(j)=j(\operatorname{resp} . \kappa(j)=j+1)(j \in \mathbf{Z} / 4 \mathbf{Z})$.

2 Corrections. We correct Theorems 4.1.1, 4.5.1 and 8.4.3 of [1] as follows:

(2.1) Correction of Theorem 4.1.1. We add the following relations to the statement of Theorem 4.1.1.

$(\mathrm{S} 4)(18)\left[E_{\kappa(2) \kappa(1) \kappa(0)}, E_{\kappa(0) \kappa(3) \kappa(2)}\right]=0$ and $\left[E_{\kappa(2)}, E_{\kappa(3) \kappa(2) \kappa(1)}^{(m)}\right]=0$, $\left[E_{\kappa(0)}, E_{\kappa(1) \kappa(0) \kappa(3)}^{(m)}\right]=0(m \geq 1)$ if $(\mathcal{E}, \Pi, p)$ is a usual datum of $\left(\mathrm{A}(1,1)^{(1)}\right)^{\mathcal{H}}$ type,

$(\mathrm{S} 4)(19)\left[E_{i}, E_{i+2}^{(m)}\right]=0(i=0,1, m \geq 1)$ if $(\mathcal{E}, \Pi, p)$ is an unusual datum of $\left(\mathrm{A}(1,1)^{(1)}\right)^{\mathcal{H}}$ type,

$(\mathrm{S} 5)(b)(b=18,19)$ The same relations with $E_{r}$ 's in place of $F_{r}$ 's in $(\mathrm{S} 4)(b)$

Proof of the corrected statement of Theorem 4.1.1. If $(\mathcal{E}, \Pi, p)$ is not of $\left(\mathrm{A}(1,1)^{(1)}\right)^{\mathcal{H}}$ type, use the original proof. Otherwise use Theorem 3.

(2.2) Correction of Theorem 4.5.1. We add the relations (S4)(b), (S5)(b) $(b=18,19)$ to the statement of Theorem 4.5.1.

The corrected statement follows immediately from Theorem 3.5.1 and the corrected Theorem 4.1.1.

(2.3) Correction of Theorem 8.4.3. We add the following relations to the statement of Theorem 8.4.3.

$(\mathrm{QS} 4)(18) Z_{\kappa(0)}\left(\left[T_{\omega_{3}}\left(K_{3}^{-1} F_{3}\right), T_{\omega_{1}}\left(K_{1}^{-1} F_{1}\right)\right]\right)=0$ and $Z_{\kappa(0)}\left(\left[E_{0}, T_{\omega_{2}}^{m+1}\right.\right.$ $\left.\left.\left(K_{2}^{-1} F_{2}\right)\right]\right)=0, Z_{\kappa(2)}\left(\left[E_{0}, T_{\omega_{2}}^{m+1}\left(K_{2}^{-1} F_{2}\right)\right]\right)=0(m \geq 1)$ if $(\mathcal{E}, \Pi, p)$ is a usual datum of $\left(\mathrm{A}(1,1)^{(1)}\right)^{\mathcal{H}}$ type,

$(\mathrm{QS} 4)(19)\left[E_{i}, T_{\omega_{i+2}}^{m+1}\left(K_{i+2}^{-1} F_{i+2}\right)\right]=0(i=0,1, m \geq 1)$ if $(\mathcal{E}, \Pi, p)$ is an unusual datum of $\left(\mathrm{A}(1,1)^{(1)}\right)^{\mathcal{H}}$ type,

$(\mathrm{QS5})(b)(b=18,19)$ The same relations with $E_{r}$ 's in place of $F_{r}$ 's in $(\mathrm{QS} 4)(b)$.

Using Proposition 8.4.2 and using $T_{\omega_{i}}$ 's, the corrected statement is obtained by exactly the same argument as in the proof of Theorem 6.8.2.

Remark 4. Let $\mathcal{D}$ be the finite dimensional Lie superalgebra such that $\operatorname{dim} \mathcal{D}=\operatorname{dim} \mathrm{D}(2,1 ; x)$ and such that its defining relations are obtained from those of $\mathrm{D}(2,1 ; x)$ by substituting -1 for $x$. Then there exists an epimorphism 
$\mathcal{D} \rightarrow \operatorname{sl}(2,2)$. (See also [2].) Let $L(\mathcal{D}, 1)$ be the infinite dimensional Lie superalgebra obtained from $\mathcal{D}$ by the same way as in Subsection 1.1. We can utilize $L(\mathcal{D}, 1)$ for giving concrete bases of $\mathcal{G}^{\star}$ and $\mathcal{G}^{\bullet}$. After doing so, we will see that the elements in the statement of Theorem 3 are indeed non-zero, which implies that the relations of the corrected statements are necessary.

\section{Acknowledgement}

We thank Y. Koga for a critical comment.

\section{References}

[1] Yamane, H., On defining relations of affine Lie superalgebras and affine quantized universal enveloping superalgebras, Publ. RIMS, Kyoto Univ., 35 (1999), 321-390.

[2] Iohara, K. and Koga, Y., Central extension of Lie superalgebras, Comment. Math. Helv., 76 (2001), 110-154. 